\title{
Sidelobe Reduction for Synthetic Aperture Focusing in High-Frequency Ultrasonic Imaging
}

\author{
Meng-Lin Li, Wei-Jung Guan and Pai-Chi Li \\ Department of Electrical Engineering, National Taiwan University, \\ Taipei, Taiwan, R. O. C.
}

Abstract - Synthetic aperture focusing with virtual sources has been previously employed to increase the penetration and to extend the depth of focus in high-frequency ultrasonic imaging, but the performance is limited by its high sidelobes. This paper introduces an adaptive weighting technique based on a focusing-quality index to suppress the sidelobes. The proposed technique is of particular value in high-frequency ultrasound where dynamic focusing using array transducers is not yet possible. Experimental data from a $50-\mathrm{MHz}$ imaging system with a single-crystal transducer are used to demonstrate the efficacy of the proposed technique on both wire targets and speckle-generating objects. Both $50-\mathrm{MHz}$ fundamental imaging and $50-\mathrm{MHz}$ tissue harmonic imaging are tested. In vivo experiments were also performed on a mouse to further demonstrate the effectiveness. The principles, and implementation issues of the new technique are also described.

\section{INTRODUCTION}

Specialized high-frequency ultrasonic imaging systems have been recently developed for the imaging of small-scale superficial structures such as the skin, the anterior chamber of the eye, and mouse embryos [1]. High-frequency ultrasound has shown promise for clinical use, but several limitations exist. The major problem is tissue attenuation limiting the penetration and reducing the signal-to-noise ratio. Moreover, high-frequency array transducers are still under development, and hence a mechanically scanned, single-crystal transducer with a fixed focus is typically used. Consequently, the image quality is significantly deteriorated in the out-of-focus region.

To improve the image quality of a fixed-focus imaging system, Passman and Ermert proposed a synthetic aperture focusing technique (SAFT) with a virtual source element [2]. The technique increases the penetration and extends the limited depth of focus of a strongly focused transducer. However, the performance of synthetic aperture focusing is limited by its high sidelobes [3]. Synthetic aperture processing treats the transducer's geometric focus as a virtual source that is assumed to produce an approximately spherical wavefront over a limited angular extent [2][3]. The fixed-focus single-crystal transducer is mechanically scanned to acquire a line of data at each scan position. Then, the SAFT is performed by appropriately delaying and summing the scan lines using the virtual-source concept.

In this paper, an adaptive weighting technique previously developed for array beamforming is extended to SAFT. The adaptive weighting for SAFT is based on a focusing-quality index, similar to the generalized coherence factor (GCF) described in [4]; here it is employed to reduce the sidelobes of SAFT. Experimental data was acquired using a 50-MHz ultrasonic imaging system and a single-element transducer with f-number $=2$. The efficacy of the proposed technique on both wire targets and diffuse scatterers is tested. The effects on contrast resolution and the background noise level are also explored.

\section{THE ADAPTIVE WEIGHTING TECHNIQUE}

The GCF adaptive weighting technique previously proposed for array beam formation is extended to SAFT here. For SAFT with a virtual source, the weighting factor is derived from the spatial spectrum of the delayed scan-line data along the scan direction (i.e., the synthetic aperture direction), which is the data received by the single-element transducer at each scan position after the focusing delays of the corresponding virtual source are applied prior to beam summation.

Fig. 1 shows simulated delayed baseband scan-line data and the associated spectra along the scan direction. A 50-MHz single-crystal transducer with a diameter of $6 \mathrm{~mm}$ and a geometric focus at $12 \mathrm{~mm}$ was simulated. The top panels in Fig. 1 present the case where a point target was in the direction of the synthesized beam, located $1.2 \mathrm{~mm}$ in front of the focal point. Fig. 1(a) shows the amplitude of the delayed baseband scan lines. It can be seen that the scan lines are generally in 
phase (i.e., horizontal wavefront), but the amplitude variations are large since the point target was illuminated by an unfocused beam. Fig. 1(b) shows the corresponding 1-D Fourier transform of the delayed scan lines along the scan direction at each range. Fig. 1(c) is the projected spectrum of Fig. 1(b). Though the data along the scan direction exhibit amplitude variations, the spectrum energy is primarily concentrated in the low-frequency region near dc.
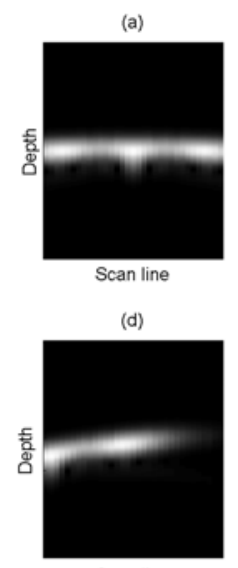

Scan line

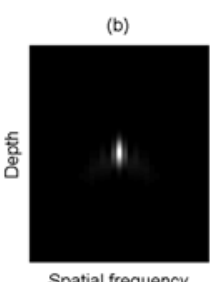
Spatial frequency

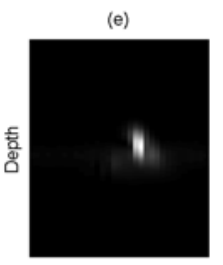

Spatial frequency
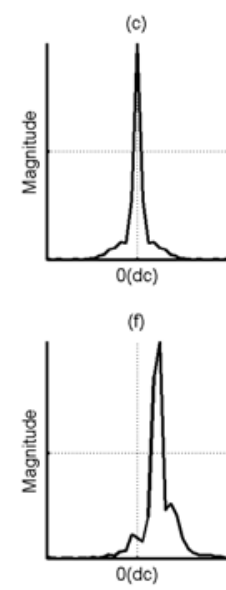

Fig. 1 Delayed baseband scan-line data and the associated spectra along the scan direction for a point target

The bottom panels of Fig. 1 show the case where the point target was off the synthesized beam axis. This corresponds to a steering error of SAFT. Fig. 1(d) shows that the delayed data are no longer in phase (i.e., tilted wavefront). The scan-line data at each range are modulated. Hence, a significant portion of the corresponding spectrum energy along the scan direction is distributed in the higher frequency region of the spectrum, as shown in Figs. 1(e) and (f). In this case, the contribution of the off-axis target to the primary beam direction needs to be minimized.

Fig. 1 shows that the spectrum of the delayed scan-line data can be used to determine the focusing quality of SAFT. The focusing quality of SAFT is directly related to the ratio of the energy in the low-frequency region (around dc) to the total energy. Hence, such an energy ratio (ER) can be used as a focusing-quality index for SAFT, and it is similar to the GCF described in [4]. The ER at a given depth can be expressed as

$$
\operatorname{ER}(t)=\frac{\sum_{k=-M_{0}}^{M_{0}}|p(k, t)|^{2}}{\sum_{k=-N / 2}^{N / 2-1}|p(k, t)|^{2}},
$$

where $p(k, t)$ is the $N$-point discrete Fourier spectrum of the delayed scan-line data along the scan direction, $N$ is the total number of scan lines included in the SAFT sum, and $k$ is the spatial frequency index. The low-frequency region is specified by a cutoff frequency $M_{0}$ in the spatial frequency index. The procedure of ER calculation is illustrated in Fig. 2. Note that an $M_{0}$ of zero means that the low-frequency range used to estimate ER is restricted to dc only.

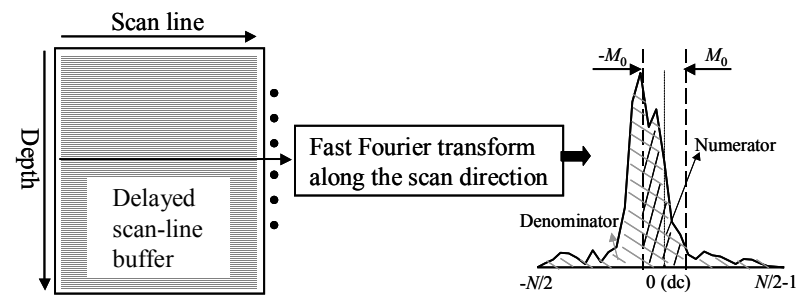

Fig. 2 Schematic diagram showing how the ER is calculated

A high ER indicates that the image object is in the direction of the synthesized beam, and thus the image intensity should be maintained. A low $\mathrm{ER}$, on the other hand, should be used to reduce the contribution of sidelobes from the outside objects to the image intensity. Hence, SAFT image quality can be improved by developing an adaptive weighting technique based on this property. The ER-weighted signal $S_{\text {weighted }}(t)$ of the SAFT signal $S_{\mathrm{SAFT}}(t)$ at a given range can be expressed as

$$
S_{\text {weighted }}(t)=\operatorname{ER}(t) \cdot S_{\mathrm{SAFT}}(t) \text {. }
$$

Note that for each beam, the weighting needs to be calculated and applied at each imaging depth.

A system block diagram of the adaptive weighting technique for SAFT is shown in Fig. 3. A baseband SAFT is implemented in this paper to reduce the data sampling requirements associated with a high-frequency system. In addition, the transducer is linearly translated along the scan direction during data acquisition. The echo signal at each scan position is received and digitized by an analog-to-digital converter, and then the received RF data are demodulated down to baseband and stored in the scan-line buffer. After $N$ scan lines are collected, appropriate virtual-source time delays and phase rotations are applied before the data are sent to the delayed scan-line buffer. The ER is then estimated using the FFT of the delayed baseband scan-line data (note that ER should be calculated at all depth points). The dc component of the spectrum (i.e., the beam sum) is then weighted by the ER, by multiplying the amplitude of the beam sum data by the corresponding ER on a point-by-point basis. The weighted data are then sent to the beam buffer for further signal processing and display. 

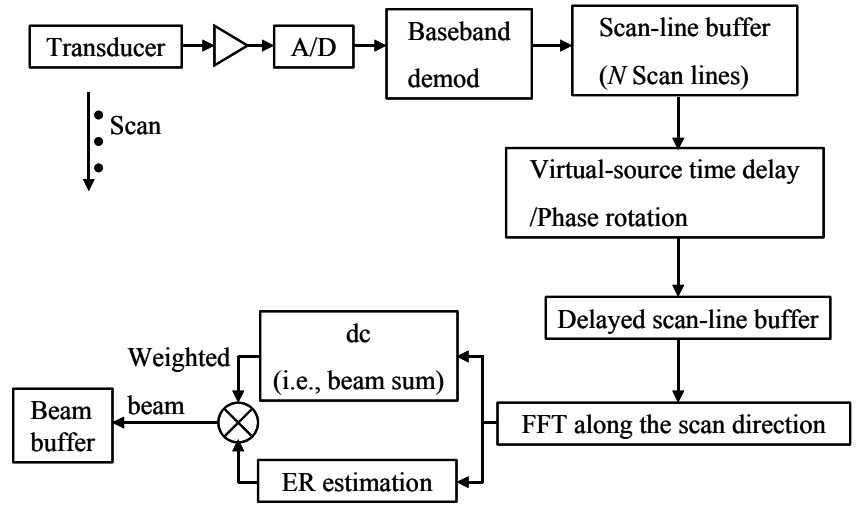

Fig. 3 System block diagram of the adaptive weighting technique for SAFT.

\section{EXPERIMENTAL RESULTS}

Experiments were conducted to investigate the efficacy of the adaptive weighting technique on sidelobe reduction in SAFT imaging. A lithium-niobate focused transducer (NIH Resource Center for Medical Ultrasonic Transducer Technology, Penn State University) was used. The transducer's center frequency is $45 \mathrm{MHz}$ of $55 \%-6$ $\mathrm{dB}$ fractional bandwidth. The transducer has a diameter of $6 \mathrm{~mm}$ and is geometrically focused at $12 \mathrm{~mm}$. The received signal was sampled with an analog-to-digital converter operating at 500 Msamples/s and 8-bit resolution.

The maximum number of scan lines for beam synthesis was limited to 32 for all the following cases, although the actual number of available scan lines may have been larger. This limitation reduced the implementation complexity of SAFT, and sped up the ER calculation. The sound velocity was assumed to be $1.48 \mathrm{~mm} / \mu \mathrm{s} . M_{0}=1$ and $M_{0}=3$ were used to calculate the ER for wire targets and speckle-generating targets, respectively. All images were displayed with a 55-dB dynamic range.

\section{A. Wire Target}

The wire target comprised a nylon wire with a diameter of $52 \mu \mathrm{m}$. Fig. 4 shows the results when the wire target was located $0.6 \mathrm{~mm}$ beyond the focal depth. A $50-\mathrm{MHz}$ Gaussian pulse was transmitted. Figs. 4(a), (b), and (c) show the original, SAFT, and SAFT-plus-weighting images, respectively. Although SAFT improves the image quality over the original image, the sidelobe level of the SAFT image is still high. This high sidelobe level is noticeably suppressed by the adaptive weighting technique. Note that the image background noise is also reduced after adaptive weighting. Figs. 4(d) and (e) show the axial and lateral projections of the images shown in Figs. 4(a), (b), and (c). The sidelobe level of the SAFT

image is suppressed more than $10 \mathrm{~dB}$ after weighting. The axial projection shows that the noise floor of the weighted image is about $8 \mathrm{~dB}$ lower than that of the SAFT image.
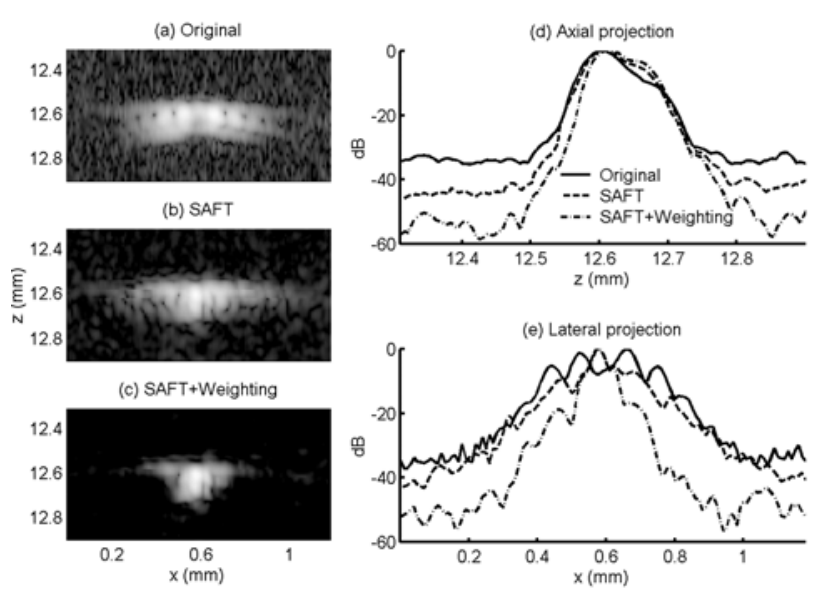

Fig. 4 Experimental results for a wire target located $0.6 \mathrm{~mm}$ beyond the focal depth

\section{B. Anechoic Cyst}

A gelatin-based phantom was also constructed and imaged to demonstrate the efficacy of the adaptive weighting technique on improving contrast resolution. The gelatin-based phantom had a $500 \mu \mathrm{m}$ anechoic region (a cyst) at its center and graphite powder $(1-2 \mu \mathrm{m})$ distributed uniformly in the background. A $50-\mathrm{MHz}$ Gaussian pulse was transmitted. Figs. 5(a), (b), and (c) show the original, SAFT, and SAFT-plus-weighting images, respectively. The center of the cyst was located $1.15 \mathrm{~mm}$ beyond the focal depth. Fig. 5 shows that detection of the cyst is noticeably improved after the adaptive weighting technique: the weighted image is less "filled in" in the cyst region than the SAFT image.

The contrast-to-noise ratio (CNR) is employed to quantitatively evaluate the improvement in contrast resolution. The CNR is calculated by taking the ratio of the image contrast to the standard deviation of image intensity in the background region, where the contrast is defined as the difference in decibels between mean intensity values in the background and the cyst region. Note that the background and cyst regions are indicated by the left- and right-hand white boxes in Fig. 5(c), respectively. The weighted image has $32 \% \mathrm{CNR}$ improvement over the SAFT image.

(a) Original

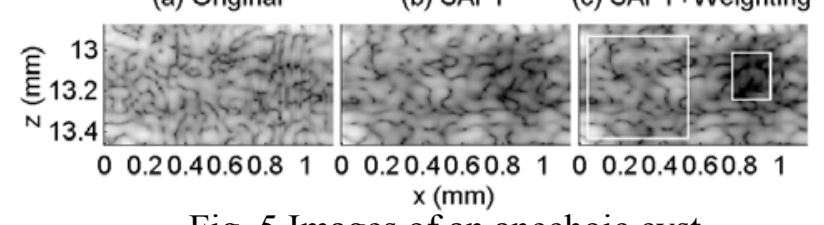

Fig. 5 Images of an anechoic cyst 


\section{50-MHz Second Harmonic Imaging}

The effectiveness of the adaptive weighting technique on tissue second harmonic imaging was also tested. In this case, a $25-\mathrm{MHz}$ pulse was used on transmit, and the received second-harmonic signal at $50 \mathrm{MHz}$ was extracted by the pulse-inversion technique which can reduce harmonic leakage resulting from the transmit waveform and the system nonlinearities. According to [5], the focal depth of the second-harmonic signal at such high frequencies is close to that of the fundamental signal at the same frequency. Hence, positions of the virtual sources for $50-\mathrm{MHz}$ second-harmonic imaging are assumed to be the same as those for $50-\mathrm{MHz}$ fundamental imaging. Fig. 6 shows the harmonic images where the wire target was located $0.69 \mathrm{~mm}$ beyond the focal depth. This figure has the same format as Fig. 4. It is clearly shown that the adaptive weighting technique is also effective for tissue harmonic SAFT imaging, with the image background noise being also significantly reduced.
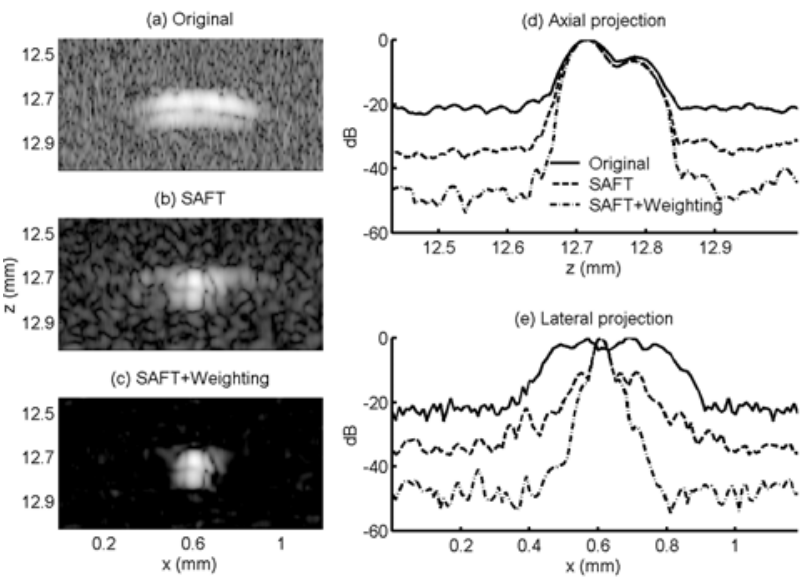

Fig. 6 50-MHz second-harmonic experimental results for a wire target located $0.69 \mathrm{~mm}$ beyond the focal depth.

\section{In Vivo Imaging of a Mouse}

An in vivo experiment on a mouse was also performed. Figs. 7(a), (b), and (c) show the 25-MHz original, SAFT, and SAFT-plus-weighting kidney images, respectively. Figs. 8(a), (b), and (c) show the $40-\mathrm{MHz}$ original, SAFT, and SAFT-plus-weighting embryo images, respectively. The weighted images in Fig. 7(c) and Fig. 8(c) exhibit superior edge definition and sharper contrast than the SAFT images in Fig. 7(b) and Fig. 8(b) over the entire depth of imaging field. Again, the image background noise is also suppressed by adaptive weighting.

\section{CONCLUSIONS}

The adaptive weighting technique using the
GCF was previously developed for array beam formation to reduce the focusing errors resulting from sound-velocity inhomogeneities. In this paper, the adaptive weighting technique was extended to reduce the sidelobes of the SAFT radiation pattern, and was applied successfully to high-frequency ultrasound where dynamic focusing using arrays is not yet feasible. Experimental results from a $50-\mathrm{MHz}$ imaging system demonstrate the effectiveness of the adaptive weighting technique in both fundamental and tissue harmonic imaging. The sidelobe level was effectively suppressed and contrast resolution was significantly improved. In addition, the image background noise was lower after the weighting. Finally, it is preferable for the proposed technique to be implemented with a baseband SAFT in order to reduce the requirement for high sampling rates associated with high-frequency systems.

\section{REFERENCES}

[1] F. S. Foster, C. J. Pavlin, and K. A. Harasiewicz et al., "Advances in ultrasound biomicroscopy," Ultrasound Med. Biol., vol. 26, no. 1, pp. 1-27, 2000.

[2] C. Passmann and H. Ermert, "A 100-MHz ultrasound imaging system for dermatologic and ophthalmologic diagnostics," IEEE Trans. Ultrason., Ferroelect., Freq. Contr., vol. 43, no. 4, pp. 545-552, 1996.

[3] G. H. Frazier and W. D. O'Brien, "Synthetic aperture technique with a virtual source element," IEEE Trans. Ultrason., Ferroelect., Freq. Contr., vol. 45, no. 1, pp. 196-207, 1998.

[4] P.-C. Li and M.-L. Li, "Adaptive imaging using the generalized coherence factor," IEEE Trans. Ultrason., Ferroelect., Freq. Contr, vol. 50, no. 2, pp. 128-141, 2003.

[5] E. Cherin, J. K. Poulsen, and A. F. W. Van der Steen et al., "Comparison of nonlinear and linear imaging techniques at high frequency," in Proc. IEEE Ultrason. Symp., 2000, pp. 1639-1643.

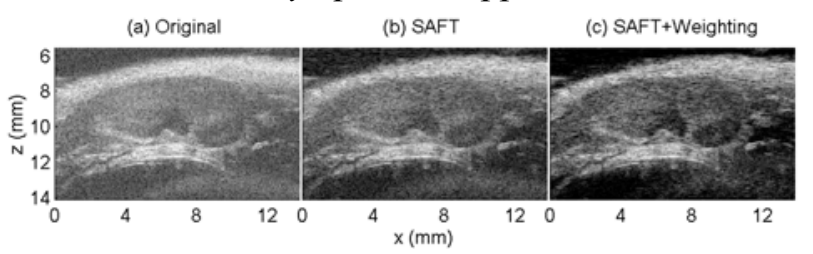

Fig. 7 25-MHz in vivo imaging of a mouse kidney

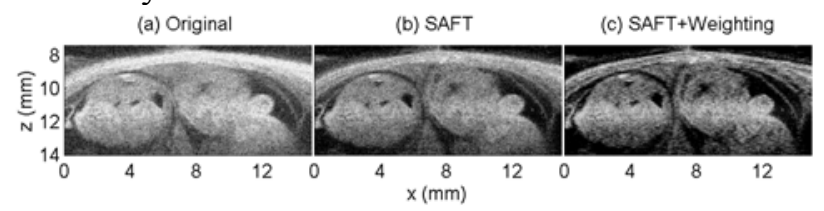

Fig. $840-\mathrm{MHz}$ in vivo imaging of mouse embryos 